\title{
Regulation and Tracking of Two-Level Quantum Systems using Measurement Feedback
}

\author{
M. Yanagisawa, A. N. Korotkov and M. R. James
}

\begin{abstract}
Two-level quantum systems are important quantum models that are useful for the conceptual development and construction of quantum technologies. In this paper we provide measurement feedback controllers for the regulation of two-level systems to pure states in the $x z$ qubit plane, and the tracking control with respect to a class of reference signals.
\end{abstract}

\section{INTRODUCTION}

The fundamental properties of two-level systems, or qubits, are studied because they are useful physical models that are mathematically simple. To use qubits for quantum technologies, we need to prepare the system in a desired state in advance. Quantum feedback control is one of the methods that may be used for state preparation. In particular, it is often of interest to prepare spin systems in eigenstates of spin operators). Measurement feedback has been used for this purpose in several ways, see [10], [9], [4], [11].

If we control the system to be in one of eigenstates of a measured observable, the state preparation results in exponential decay of the mean square error to zero. However, when the system is driven to a state that is not an eigenstate of the measured observable, the qubit is constantly influenced by measurement noise so while the mean square error remains mean-square bounded, it does not tend to zero. This means that such a desired state cannot be prepared with probability one [7], [5]. In this case, it is important to quantify the behavior of the error.

In general, the strength of the measurement noise is determined by a coupling constant to the measurement device. Thus, it is expected that the error probability will depend on the coupling constant. Meanwhile, if feedback is applied, the error will be reduced due to the noise reduction effect of feedback.

The purpose of this paper is to investigate the problem of regulation to states that are not eigenstates of the measured observable. We design a regulating measurement feedback control law and provide explicit bounds on the mean square error. We also investigate a tracking problem where the measurement feedback controller is designed to follow a given reference signal.

We begin in section II by describing qubits and the Bloch sphere, recalling the quantum filter for the problem we

M. Yanagisawa is with Department of Engineering, The Australian National University, Canberra, ACT 0200, Australia y . m@a nu . edu • au

A. N. Korotkov is with Department of Electrical Engineering, University of California, Riverside, CA, 92521, USA korotkov@ee.ucr.edu

M. R. James is with Department of Engineering, The Australian National University, Canberra, ACT 0200, Australia Mat thew. James a anu. edu. au consider, and also recalling a basic stochastic stability result. Section III contains our results on regulation, while tracking is considered in section IV. Finally, the effect of decoherence is discussed in section $\mathrm{V}$.

\section{Preliminaries}

\section{A. Qubits}

A qubit is a two dimensional quantum system defined on the Hilbert space $\mathbf{C}^{2}$, the two-dimensional complex vector space. Physical observables are represented as operators on this Hilbert space, and are given by $2 \times 2$ Hermitian matrices. Qubit states are described by density operators $\rho$, which may be represented explicitly in the form

$$
\rho=\frac{1}{2}\left(I+x \sigma_{x}+y \sigma_{y}+z \sigma_{z}\right),
$$

where $I$ is the identity matrix, $x^{2}+y^{2}+z^{2} \leq 1$, and

$$
\begin{aligned}
\sigma_{x} & =\left[\begin{array}{ll}
0 & 1 \\
1 & 0
\end{array}\right], \\
\sigma_{y} & =\left[\begin{array}{cc}
0 & \mathrm{i} \\
-\mathrm{i} & 0
\end{array}\right], \\
\sigma_{z} & =\left[\begin{array}{cc}
1 & 0 \\
0 & -1
\end{array}\right],
\end{aligned}
$$

are the Pauli matrices (these operators are the basis of the Lie algebra $s u(2)$ and the corresponding Lie group represents the rotation of the qubit about the $x, y$ and $z$ axis, respectively). It is not difficult to see that if $x^{2}+y^{2}+z^{2}=1$, then the rank of $\rho$ is one. In this case, the state is called pure, and otherwise mixed. From the parameterization above, the qubit is identified with a vector $(x, y, z)$ whose elements can be thought of as the $x, y$ and $z$ elements of the qubit, respectively. A vector $(x, y, z)$ is called a Bloch vector and is used to represent the density operator.

\section{B. Quantum filter}

We consider a qubit that is continuously monitored. The measurement signal may be used for feedback control. Indeed, we suppose that the spin observable $\sigma_{z}$ is measured through a channel with coupling strength $k$. The measurement signal $\zeta_{t}$ (which corresponds to a rescaled and intergrated physical signal) is given by $d \zeta_{t}=k \operatorname{tr}\left[\rho_{t} \sigma_{z}\right] d t+d w_{t}$, where $\rho_{t}$ is the conditional state and $w_{t}$ is the innovation process (a standard Wiener process). We also suppose that the qubit is subject to decoherence (pure dephasing) coupled to a noise channel via $\sigma_{z}$ with strength parameter $\gamma$. The 
evolution of the conditional density matrix given the measurement outcome is described by the quantum filter (also called stochastic master equation) [2], [16], [3], [6],

$$
\begin{aligned}
d \rho= & \left(\mathrm{i}[H, \rho]-\frac{k^{2}}{8}\left[\sigma_{z},\left[\sigma_{z}, \rho\right]\right]-\frac{\gamma}{4}\left[\sigma_{z},\left[\sigma_{z}, \rho\right]\right]\right) d t \\
& +\frac{k}{2}\left\{\left(\sigma_{z} \rho+\rho \sigma_{z}\right)-2 \rho \operatorname{Tr}\left(\rho \sigma_{z}\right)\right\}\left(d \zeta_{t}-k \operatorname{tr}\left[\rho_{t} \sigma_{z}\right] d t\right) .
\end{aligned}
$$

Here we interpret stochastic differential equations in the Ito sense. $H$ in (5) is the qubit Hamiltonian that may be modulated by external classical input signals. We assume that the Hamiltonian is of the form

$$
H=\frac{h}{2} \sigma_{y}+\frac{g}{2} \sigma_{z},
$$

where $h$ and $g$ are control signals to be designed.

Let us rewrite the stochastic master equation in terms of the Bloch vector. From (1) and (5), we have

$$
\begin{aligned}
d\left[\begin{array}{l}
x \\
y \\
z
\end{array}\right]= & {\left[\begin{array}{ccc}
-\frac{k^{2}}{2}-\gamma & g & h \\
-g & -\frac{k^{2}}{2}-\gamma & 0 \\
-h & 0 & 0
\end{array}\right]\left[\begin{array}{l}
x \\
y \\
z
\end{array}\right] d t } \\
& +k\left[\begin{array}{c}
-x z \\
-y z \\
1-z^{2}
\end{array}\right]\left(d \zeta_{t}-k \operatorname{tr}\left[\rho_{t} \sigma_{z}\right] d t\right) .
\end{aligned}
$$

This is the system to be controlled. The diagonal elements of the first matrix represent decoherence, and off-diagonal elements are rotations due to control. The control input signals $h$ and $g$ depend causally on the measurement signal $\zeta_{t}$. Assuming the initial state $\rho_{0}$ is known, this may be achieved by implementing the quantum filter (7) and driving the filter by the measurement signal $\zeta_{t}$. The input signals $h$ and $g$ may be chosen to depend on the Bloch vector $(x, y, z)$ for the conditional state computed by the quantum filter (7). This type of measurement feedback is based on feedback of the conditional state, and is thus dynamic in nature.

We remark that (5) or (7) is a model for the physical system being controlled, and if all parameters and the initial condition are known, this model will coincide with the quantum filter used in the implementation of the controller, the later being implemented, say, in a computer algorithm.

\section{Stochastic stability}

In this section, we give a well-known result on stochastic differential equations [14] for the purpose of the spin control problem.

Let us consider a stochastic differential equation

$$
d \xi=a(\xi) d t+b(\xi) d w
$$

where $w$ is a standard Wiener process. The infinitesimal generator for the process $\xi_{t}$ is given by

$$
\mathcal{L}=\mathcal{L}_{D}+\mathcal{L}_{S},
$$

where

$$
\begin{aligned}
& \mathcal{L}_{D}=a(\xi) \frac{\partial}{\partial \xi}, \\
& \mathcal{L}_{S}=\frac{b^{2}(\xi)}{2} \frac{\partial^{2}}{\partial \xi^{2}} .
\end{aligned}
$$

Lemma 1: Assume that there exists a function $V(\xi)$ such that for positive constants $\alpha$ and $\beta$,

$$
\mathcal{L} V \leq-\alpha V+\beta .
$$

Then, the function $V$ is bounded as follows:

$$
E\left[V\left(\xi_{t}\right)\right] \leq e^{-\alpha t} E\left[V\left(\xi_{0}\right)\right]+\frac{\beta}{\alpha}\left(1-e^{-\alpha t}\right) .
$$

Proof: Integrating equation (8) and taking expectations we find that

$$
E\left[V\left(\xi_{t+h}\right)-V\left(\xi_{t}\right)+\alpha \int_{t}^{t+h} V\left(\xi_{r}\right) d r\right] \leq \beta h .
$$

This implies

$$
\frac{d}{d t} E\left[V\left(\xi_{t}\right)\right] \leq-\alpha E\left[V\left(\xi_{t}\right)\right]+\beta,
$$

from which (12) follows.

If the system has a static equilibrium at point, say $\xi=0$, i.e., $a(0)=b(0)=0$, and the hypotheses of Lemma 1 are satisfied with $\beta=0$ for a positive definite function $V(\xi) \geq c|\xi|^{2}(c>0)$, then Lemma 1 implies $\left|\xi_{t}\right|$ decays exponentially to zero, and this rate of convergence is deterministic. We refer to such cases as (exponentially) asymptotically stable in mean square. On the other hand, if stable in mean square, $b(0) \neq 0$, then the system is constantly disturbed by the stochastic noise and we have $\beta \neq 0$ in general. In this case the trajectory remains meansquare bounded (by a deterministic bound) but does not tend to zero asymptotically. For a controlled system of the form (8), it is important that the term $\beta / \alpha$ be made as small as possible by appropriate choice of feedback control.

\section{REGULATION}

In this section we assume that there is no decoherence in the system. As will be discussed later (Sec. V), in this case any initial state becomes pure asymptotically, so here we consider a control problem only for pure states (the length of the spin is always maximal, i.e., $x^{2}+y^{2}+z^{2}=1$ ). The purpose of the control is to drive the spin to an arbitrary point on the Bloch sphere in the $x z$-plane. It is known that we can drive the spin to the spin-up or spin-down state with probability 1 . This is because these states are the eigenstates of the measured observable $\sigma_{z}$, i.e., static equilibrium points of (7). As a result, once the spin reaches one of these two points, it is fixed there due to the projection effect of the measurement. On the other hand, if we control the spin to non-equilibrium points, its probability cannot be one because the spin is subject to fluctuation. We first consider the design of the control input, and then, give the error probability around the target point. 


\section{A. Description of pure states}

In this section, we assume that $\gamma=0$ in (7), which means that there is no additional decoherence in the system (the case of $\gamma \neq 0$ will be considered later). We set $g=0$; then by writing an equation for $d\left(y^{2}\right)$ from (7) it is possible to show that the $y$ coordinate of the Bloch vector decays to zero (at least as a power-law, and exponentially if $x^{2} \geq$ const $>0$ ). In what follows we assume this has occurred.

For a pure state in the $x z$-plane, the Bloch vector is represented in polar coordinates as

$$
\begin{aligned}
& x=\sin \theta, \\
& z=\cos \theta .
\end{aligned}
$$

Then the system is described in these coordinates as

$$
d \theta=\left[h-\frac{k^{2}}{2} \sin \theta \cos \theta\right] d t-k \sin \theta d w,
$$

where the innovation process (a standard Weiner process) is given by

$$
d w_{t}=d \zeta_{t}-k \operatorname{tr}\left[\rho_{t} \sigma_{z}\right] d t
$$

Our purpose is to drive the spin to a point on the Bloch sphere in the $x z$-plane $\theta=\theta_{0}$ by designing $h(\theta)$. Let us introduce a transform

$$
\xi=\theta-\theta_{0} .
$$

Then, the system is expressed as

$$
d \xi=\left[h(\xi)-\frac{k^{2}}{4} \sin 2\left(\xi+\theta_{0}\right)\right] d t-k \sin \left(\xi+\theta_{0}\right) d w .
$$

Since there is no static equilibrium for $\theta_{0} \neq 0$, no control can achieve exponential decay to zero of the mean square error. Our purpose is now to reduce the deviation of the Bloch vector from the desired angle $\theta_{0}$.

\section{B. Feedback law}

To control the Bloch vector, we consider a control input of the form

$$
h(\xi)=-h_{0} \sin \frac{\xi}{2},
$$

where $h_{0}$ is a positive constant (notice a discontinuity of the control at $\xi= \pm \pi$ ).

Theorem 1: Consider a two level system described by (16). If we use a control input (17) with $h_{0}>k^{2}$, then we have

$$
\begin{aligned}
\mathrm{E}\left[1-\cos \frac{\xi_{t}}{2}\right] \leq & e^{-\left(h_{0}-k^{2}\right) t / 2} \mathrm{E}\left[1-\cos \frac{\xi(0)}{2}\right] \\
& +\frac{k^{2}}{2\left(h_{0}-k^{2}\right)}\left[\sin ^{2} \theta_{0}+\frac{1}{2}\left|\sin 2 \theta_{0}\right|\right] \\
& \times\left[1-e^{-\left(h_{0}-k^{2}\right) t / 2}\right] .
\end{aligned}
$$

Proof: For the system (16) with feedback (17), the infinitesimal generators (10) are given as

$$
\begin{aligned}
\mathcal{L}_{D} & =\left[-h_{0} \sin \frac{\xi}{2}-\frac{k^{2}}{4} \sin 2\left(\xi+\theta_{0}\right)\right] \frac{\partial}{\partial \xi}, \\
\mathcal{L}_{S} & =\frac{1}{2}\left[k \sin \left(\xi+\theta_{0}\right)\right]^{2} \frac{\partial^{2}}{\partial \xi^{2}} .
\end{aligned}
$$

Let

$$
V=1-\cos \frac{\xi}{2}
$$

Then, we have

$$
\begin{aligned}
\left(\mathcal{L}_{D}+\mathcal{L}_{S}\right) V \leq & -\frac{h_{0}}{2} \sin ^{2} \frac{\xi}{2}+\frac{k^{2}}{2} \cos ^{2} \frac{\xi}{2} \sin ^{2} \frac{\xi}{2} \\
& +\frac{k^{2}}{4} \sin ^{2} \theta_{0}+\frac{k^{2}}{8}\left|\sin 2 \theta_{0}\right| .
\end{aligned}
$$

Now, we want to find $\alpha$ and $\beta$ such that

$$
\begin{aligned}
& -\frac{h_{0}}{2} \sin ^{2} \frac{\xi}{2}+\frac{k^{2}}{2} \cos ^{2} \frac{\xi}{2} \sin ^{2} \frac{\xi}{2}+\frac{k^{2}}{4} \sin ^{2} \theta_{0}+\frac{k^{2}}{8}\left|\sin 2 \theta_{0}\right| \\
& \leq-\alpha\left(1-\cos \frac{\xi}{2}\right)+\beta
\end{aligned}
$$

Let $\cos (\xi / 2)=1-\epsilon(0<\epsilon<1)$. Then, it is sufficient to find $\alpha$ and $\beta$ such that

$$
\begin{aligned}
& \frac{1}{2}\left(h_{0}-k^{2}\right) \epsilon^{2}-\left(h_{0}-k^{2}-\alpha\right) \epsilon \\
& +\frac{k^{2}}{4} \sin ^{2} \theta_{0}+\frac{k^{2}}{8}\left|\sin 2 \theta_{0}\right|-\beta \leq 0 .
\end{aligned}
$$

If $h_{0}>k^{2}$, this is satisfied when

$$
\begin{aligned}
& \frac{k^{2}}{4} \sin ^{2} \theta_{0}+\frac{k^{2}}{8}\left|\sin 2 \theta_{0}\right|-\beta \leq 0, \\
& -\frac{1}{2}\left(h_{0}-k^{2}\right)+\alpha+\frac{k^{2}}{4} \sin ^{2} \theta_{0}+\frac{k^{2}}{8}\left|\sin 2 \theta_{0}\right|-\beta \leq 0 .
\end{aligned}
$$

If $\alpha \geq\left(h_{0}-k^{2}\right) / 2$ then the ratio $\beta / \alpha$ is bounded below by

$$
\frac{k^{2}}{2\left(h_{0}-k^{2}\right)}\left(\frac{1}{2} \sin ^{2} \theta_{0}+\frac{1}{4}\left|\sin 2 \theta_{0}\right|\right) \leq \frac{\beta}{\alpha} .
$$

Equality is obtained with the choice

$$
\begin{aligned}
& \alpha=\frac{h_{0}-k^{2}}{2}, \\
& \beta=\frac{k^{2}}{4} \sin ^{2} \theta_{0}+\frac{k^{2}}{8}\left|\sin 2 \theta_{0}\right| .
\end{aligned}
$$

Now applying Lemma 1 yields the inequality (18).

From inequality (18), one can see that for large $t$

$$
\mathrm{E}\left[1-\cos \frac{\xi_{t}}{2}\right] \leq \frac{k^{2}}{2\left(h_{0}-k^{2}\right)}\left[\sin ^{2} \theta_{0}+\frac{1}{2}\left|\sin 2 \theta_{0}\right|\right],
$$

and so the feedback law (17) is stable in mean square. If the feedback gain is sufficiently large, i.e., $k^{2} \ll h_{0}$, then the system is stabilized well around the target point $\theta=$ $\theta_{0}(\xi=0)$ and the error can be sufficiently small after a long time. If this is the case, the function $V$ can be approximately expressed as

$$
V \sim \frac{\xi^{2}}{8}
$$


and the control input is

$$
h \sim-\frac{h_{0} \xi}{2},
$$

which is simply proportional to the error, as usually considered in linear stabilizing problems. Then, the mean square error can be asymptotically given as

$$
\mathrm{E}\left[\xi_{t}^{2}\right] \leq \frac{4 k^{2}}{h_{0}-k^{2}}\left[\sin ^{2} \theta_{0}+\frac{1}{2}\left|\sin 2 \theta_{0}\right|\right] .
$$

In the case $\theta_{0}=0, \pm \pi$, the asymptotic bound on the mean square error is zero. That is to say, the system can be stabilized at these two points with probability one, [10]. This is a natural result because these states are the eigenstates of the measured observable.

Even though in this paper we assume a perfect knowledge of the qubit model, let us briefly discuss what happens if we use a wrong initial state. Then Eq. (14) describes the evolution of actual angle $\theta$, while the evolution of our imperfectly known angle $\tilde{\theta}$ (as computed within the controller) is given by equation

$$
\begin{aligned}
d \tilde{\theta}= & {\left[h-\frac{k^{2}}{2} \sin \tilde{\theta} \cos \tilde{\theta}\right] d t } \\
& -k \sin \tilde{\theta}[d w+k(\cos \theta-\cos \tilde{\theta}) d t] .
\end{aligned}
$$

Therefore, the inaccuracy evolves as

$$
\begin{aligned}
& d(\tilde{\theta}-\theta)=-k^{2} \sin \frac{\tilde{\theta}-\theta}{2}\left(\cos \theta \cos \frac{\tilde{\theta}+\theta}{2}\right. \\
& \left.+\sin \tilde{\theta} \sin \frac{\tilde{\theta}+\theta}{2}\right) d t-k(\sin \tilde{\theta}-\sin \theta) d w .
\end{aligned}
$$

Brief analysis of this equation shows that small inaccuracy decreases exponentially (assuming $\sin ^{2} \theta \geq$ const $>0$ ), while evolution of an initially large inaccuracy requires a more detailed analysis. Most importantly, this shows that our feedback procedure is robust at least against small inaccuracy of the initial state.

\section{TRACKING}

In the previous section, we considered a spin control problem to stabilize at a fixed point on the Bloch sphere in the $x z$-plane. In this section, we assume that the control target is time varying in the $\theta$ coordinate. Our purpose is to drive spin to a moving target as close as possible via feedback. Suppose that the reference signal to be tracked $\theta_{0}(\cdot)$ is described by

$$
\frac{d \theta_{0}(t)}{d t}=f\left(\theta_{0}(t), t\right)
$$

Let us define an error as

$$
\xi=\theta-\theta_{0}
$$

Theorem 2: Consider an input

$$
h(\xi, t)=-h_{0} \sin \frac{\xi}{2}+f\left(\theta_{0}(t), t\right) \quad\left(h_{0}>k^{2}\right)
$$

for a two level system described by (16) and a target (35). Then, we have

$$
\begin{aligned}
\mathrm{E}\left[1-\cos \frac{\xi_{t}}{2}\right]< & e^{-\left(h_{0}-k^{2}\right) t / 2} \mathrm{E}\left[1-\cos \frac{\xi(0)}{2}\right] \\
& +\frac{k^{2} q}{2\left(h_{0}-k^{2}\right)}\left[1-e^{-\left(h_{0}-k^{2}\right) t / 2}\right],
\end{aligned}
$$

where $q \equiv \max _{t}\left(\sin ^{2} \theta_{0}(t)+\left|\sin 2 \theta_{0}(t)\right| / 2\right)$.

Proof: The error is given by

$$
\begin{aligned}
d \xi= & {\left[h(\xi, t)-\frac{k^{2}}{2} \sin \left(\xi+\theta_{0}\right) \cos \left(\xi+\theta_{0}\right)-f\left(\theta_{0}, t\right)\right] d t } \\
& -k \sin \left(\xi+\theta_{0}\right) d w .
\end{aligned}
$$

The infinitesimal generators for this system are given as

$$
\begin{aligned}
\mathcal{L}_{D}= & {\left[h(\xi, t)-\frac{k^{2}}{2} \sin \left(\xi+\theta_{0}\right) \cos \left(\xi+\theta_{0}\right)\right.} \\
& \left.-f\left(\theta_{0}, t\right)\right] \frac{\partial}{\partial \xi}, \\
\mathcal{L}_{S}= & \frac{1}{2}\left[k \sin \left(\xi+\theta_{0}\right)\right]^{2} \frac{\partial^{2}}{\partial \xi^{2}} .
\end{aligned}
$$

Let

$$
V=1-\cos \frac{\xi}{2}
$$

Then, we have

$$
\begin{aligned}
\mathcal{L} V= & h(\xi, t) \sin \xi+\frac{k^{2}}{2} \sin \left(\xi+\theta_{0}\right) \sin \theta_{0} \\
& -f\left(\theta_{0}, t\right) \sin \xi .
\end{aligned}
$$

For the input (37),

$$
\mathcal{L} V \leq-\frac{h_{0}-k^{2}}{2} \sin ^{2} \frac{\xi}{2}+\frac{k^{2} q}{4} .
$$

Now, we want to find $\alpha$ and $\beta$ such that

$$
-\frac{h_{0}-k^{2}}{2} \sin ^{2} \frac{\xi}{2}+\frac{k^{2} q}{4} \leq-\alpha\left(1-\cos \frac{\xi}{2}\right)+\beta
$$

As in the proof of Theorem 1 , the ratio $\beta / \alpha$ is bounded below as

$$
\frac{k^{2} q}{2\left(h_{0}-k^{2}\right)} \leq \frac{\beta}{\alpha},
$$

and the equality is attained when $\alpha=\left(h_{0}-k^{2}\right) / 2$. Lemma 1 yields the inequality (18).

From inequality (38), one can see that for large $t$

$$
\mathrm{E}\left[1-\cos \frac{\xi_{t}}{2}\right]<\frac{k^{2} q}{2\left(h_{0}-k^{2}\right)} .
$$

For the same reason as in the previous section, the system is driven to the target well if the feedback gain $h_{0}$ is sufficiently large, i.e., $h_{0} \gg k^{2}$. Then, the mean square error is bounded for large $t$

$$
\mathrm{E}\left[\xi_{t}^{2}\right]<\frac{4 k^{2} q}{h_{0}-k^{2}},
$$

and the control input is approximately expressed as

$$
h(\xi, t)=-\frac{h_{0} \xi}{2}+f\left(\theta_{0}(t), t\right) .
$$


If we construct a control input simply proportional to the error $\xi$ alone as we did in the previous section, i.e.,

$$
h(\xi)=-\frac{h_{0} \xi}{2},
$$

then the bound is given by

$$
\mathrm{E}\left[1-\cos \frac{\xi_{t}}{2}\right]<\frac{k^{2} q+2 r}{2\left(h_{0}-k^{2}\right)},
$$

where $r \equiv \max _{t}\left|f\left(\theta_{0}(t), t\right)\right|$. This bound is larger by the additional term depending on $r$. The system can still be well controlled by a large gain feedback. However, if the target is rapidly changing i.e., $r \gg h_{0}$, then it becomes more difficult to track the reference signal. The input of the form (37) is designed in such a way that this does not happen.

\section{INFLUENCE OF DECOHERENCE}

In this section, we consider a case where the system is subject to decohering influences, $\gamma \neq 0$. Decoherence may destroy the purity of the state, which means that the state is in the interior and not on the surface of the Bloch sphere. Thus decoherence may shorten the length of the spin state vector, and so we need to consider the time evolution of the length of the spin state vector explicitly. The recovery of the spin vector length strongly depends on the direction of the spin.

If the system is in a mixed state, it is described in $x z$-plane as

$$
\begin{aligned}
d p= & {\left[-\gamma p \sin ^{2} \theta+\frac{k^{2}}{2} \frac{1-p^{2}}{p} \sin ^{2} \theta\right] d t } \\
& +k\left(1-p^{2}\right) \cos \theta d w, \\
d \theta= & {\left[-\gamma \sin \theta \cos \theta+\frac{k^{2}}{2} \sin \theta \cos \theta \frac{2-3 p}{p^{2}}+h\right] d t } \\
& -\frac{k}{p} \sin \theta d w,
\end{aligned}
$$

where $p$ is the length of the Bloch vector (the qubit purity is usually defined as $\left.\operatorname{Tr} \rho^{2}=\left(1+p^{2}\right) / 2\right)$. For this system, the infinitesimal operators are given as

$$
\begin{aligned}
\mathcal{L}_{D}= & {\left[-\gamma p \sin ^{2} \theta+\frac{k^{2}}{2} \frac{1-p^{2}}{p} \sin ^{2} \theta\right] \frac{\partial}{\partial p} } \\
& +\left[-\gamma \sin \theta \cos \theta+\frac{k^{2}}{2} \sin \theta \cos \theta \frac{2-3 p}{p^{2}}+h\right] \frac{\partial}{\partial \theta} \\
\mathcal{L}_{S}= & \frac{k^{2}}{2}\left(1-p^{2}\right)^{2} \cos ^{2} \theta \frac{\partial^{2}}{\partial p^{2}} \\
& -\frac{k^{2}}{p}\left(1-p^{2}\right) \sin \theta \cos \theta \frac{\partial^{2}}{\partial \theta \partial p}+\frac{k^{2}}{2 p^{2}} \sin ^{2} \theta \frac{\partial^{2}}{\partial \theta^{2}}
\end{aligned}
$$

Let us consider a Lyapunov function

$$
V=\frac{p^{2}}{2} \text {. }
$$

This yields

$$
\mathcal{L} V=\frac{k^{2}}{2}\left(1-p^{2}\right)\left(1-p^{2} \cos ^{2} \theta\right)-\gamma p^{2} \sin ^{2} \theta .
$$

Note that dependence on the control input $h$ comes here only via the angle $\theta$. If the state is mixed $(p<1)$ and there is no decoherence in the system $(\gamma=0)$, then for all $\theta$

$$
\mathcal{L} V>0
$$

which indicates that the system always becomes pure asymptotically. In this case, if we want to drive the system to $(p, \theta)=\left(1, \theta_{0}\right)$ via feedback, we only need to control $\theta$ as we did in the previous sections. However, the convergence rate (purity change) of the system is dependent on $\theta$. Obviously, $\mathcal{L} V$ is the largest (for $\gamma=0$ ) when $\theta= \pm \pi / 2$ [8], [12]. On the other hand, it has been shown that the average time to reach a given purity is minimized along $\theta=0, \pi$ [13]. This $\theta$-dependency of the purity change becomes critical if $\gamma \neq 0$

In the case $\gamma \neq 0$ the purity factor $p$ becomes a fluctuating quantity. However, at small $\gamma$ it is still distributed in the vicinity of $p=1$ asymptotically, because of the first term in Eq. (56). Therefore, the results of Secs. III and IV should remain approximately valid for a small decoherence $\gamma$.

\section{CONCLUSION}

We have considered the design of feedback control to drive the spin to an arbitrary point on the Bloch sphere in the $x z$ plane. In general it is impossible to asymptotically stabilize such a point because the spin is continually influenced by the measurement noise. However, we provided bounds on the mean square deviation from the desired reference state, ensuring mean square stability of the error. In the special cases $\theta=0, \pm \pi$, the feedback control can asymptotically stabilize the spin, meaning the error tends to zero asymptotically. This technique was applied to a tracking problem where the reference signal is time varying. In this case, we can see that the reference signal model forms part of the controller. More general regulation and tracking control designs will be given in a future publication, together with detailed analysis.

In this paper we mostly considered a rather idealized case when there is no decoherence, all the system parameters and the initial state are perfectly known, and monitoring of the qubit state is also perfect. It would be important to check that our results remain approximately valid when these assumptions are slightly violated. Even though we do not prove this assertion explicitly, we are practically confident in it because of the following. Small decoherence was discussed in Sec. V and should not drastically change the results of Secs. III and IV. As was discussed in Sec. III, a small inaccuracy of the initial state decreases exponentially in the course of the feedback control. A small inaccuracy of the parameters $k$ and $h$ would lead to a slow deviation of our estimated state from the actual state (still within the $x z$ plane); however, this deviation is constantly decreased by the same mechanism as for an inaccurate initial state. Small non-zero parameter $g$ would lead to a gradually accumulating $y$-coordinate; however, the attraction to the $x z$-plane due to measurement (mentioned in Sec. III) constantly moves $y$ towards zero. As a result, our results should be robust against small imperfections of state monitoring and parameters. 


\section{REFERENCES}

[1] R. L. Hudson and K. R. Parthasarathy, Quantum Ito's formula and stochastic evolutions, Comm. Math. Phys. 93, pp. 301-323, 1984.

[2] V. P. Belavkin, Quantum stochastic calculus and quantum nonlinear filtering, J. Multivar. Anal. 42, pp. 171-201, 1992.

[3] H. M. Wiseman and G. J. Milburn, Quantum-theory of field-quadrature measurements, Phys. Rev. A 47, 642, 1993.

[4] H. F. Hofmann, G. Mahler, O. Hess, Quantum control of atomic systems by homodyne detection and feedback, Phys. Rev. A 57, pp. 4877-4888, 1998.

[5] J. Wang and H. M. Wiseman, Feedback-stabilization of an arbitrary pure state of a two-level atom, Phys. Rev. A 64, 063810, 2001.

[6] A. N. Korotkov, Selective quantum evolution of a qubit state due to continuous measurement, Phys. Rev. B 63, 115403, 2001.

[7] R. Ruskov and A. N. Korotkov, Quantum feedback control of a solidstate qubit, Phys. Rev. B 66, 041401, 2002.

[8] K. Jacobs, How to project qubits faster using quantum feedback, Phys. Rev. A 67, 030301, 2003

[9] J. K. Stockton, R. van Handel and H. Mabuchi, Deterministic Dicke state preparation with continuous measurement and control, Phys. Rev. A 70, 022106, 2004.

[10] R. van Handel, J. K. Stockton and H. Mabuchi, Feedback control of quantum state reduction, IEEE Trans. Automat. Cont. 50, pp. 768-780, 2005.

[11] M. Mirrahimi and R. van Handel, Stabilizing feedback controls for quantum systems, SIAM Journal on Control and Optimization 46 (2), pp. 445-467, 2007.

[12] J. Combes and K. Jacobs, Rapid State Reduction of Quantum Systems Using Feedback Control, Phys. Rev. Lett. 96, 010504, 2006.

[13] H. M. Wiseman and J. F. Ralph, Reconsidering rapid qubit purification by feedback, New J. Phys. 8, 90, 2006.

[14] R. Z. Khasminskii, Stochastic stability of differential equations. Sijthoff and Noordhoff, Alphen aan den Rijn, Netherlands, 1980

[15] C. W. Gardiner and P. Zoller, Quantum Noise (Springer, Heidelberg) 2004.

[16] L. Bouten and R. Van Handel and M.R. James, An introduction to quantum filtering, SIAM J. Control and Optimization 46 (6), pp. 21992241, 2007. 